\title{
SPECTRUM AND SEMIGROUPS OF ELLIPTIC OPERATORS ON HOMOGENEOUS SPACES
}

\author{
KENNETH D. JOHNSON
}

\section{Introduction and notation}

For a manifold $X$ we denote the bounded continuous real (resp. complex) valued functions on $X$ by $\mathscr{B}(X)($ resp. $\mathscr{B}(X, C))$. For $f \in \mathscr{B}(X)($ resp. $\mathscr{B}(X, C))$ we set $\|f\|=\sup _{x \in X}|f(x)|$. Let $\mathscr{B}^{n}(X)\left(\right.$ resp. $\left.\mathscr{B}^{n}(X, C)\right)$ denote the real (resp. complex) valued functions which are bounded and continuously differentiable of order $n$.

Let $G$ be a Lie group and $H$ a closed subgroup. Let $\Delta$ be a second order elliptic differential operator on $G / H$, which is invariant under the left action of $G$. Assume that in any local coordinate neighborhood $\Delta$ is of the form:

$$
a_{i j}(x) \frac{\partial^{2}}{\partial x^{i} \partial x^{j}}+b_{k}(x) \frac{\partial}{\partial x^{k}},
$$

where $\left(a_{i j}(x)\right)$ is a positive definite symmetric matrix and the $b_{k}(x)$ 's are real. (We use Einstein summation convention.) Unless otherwise stated we will assume $G, H$ and $\Delta$ as above.

In $\S 2$, we show that $\Delta$ generates a continuous semigroup of probablility measures on $\beta(G / H)$, the Stone-Čech compactification of $G / H$. This extends a result of Hunt [3]. We also obtain restrictions on the spectrum of $\Delta$. If, moreover, $G / H$ admits a $G$-invariant Riemannian metric and $\Delta$ is the Laplacian of this metric, then the above results are strengthened.

Throughout this paper, the crucial technique is given by Lemma 2.1 which has been proven by Omori [4] under somewhat different circumstances.

This paper is developed from the author's thesis. The author would like to thank his advisor Joseph A. Wolf for his constant encouragement, and would also like to thank the referee for his many helpful suggestions.

\section{Spectrum and semigroup}

Lemma 2.1. Let $f$ be a real valued $C^{2}$-function on $G / H$, which is bounded from above. Then for an arbitrarily fixed point $p$ of $G / H$ and for any $\varepsilon>0$,

Received February 9, 1970, and, in revised forms, September 29, 1970, December 4, 1970, and March 9, 1971. 
there exists a q depending on puch that: (i) $f(q) \geq f(p)$, and (ii) $(\Delta f)(q)<\varepsilon$.

Proof. Let $b=\sup f$. Select $\varphi \in \mathscr{B}^{2}(G / H)$ such that $\varphi \geq 0,\|\Delta \varphi\|<\varepsilon$, $\varphi(e H)>0$ and $\operatorname{supp} \varphi=C$ is compact. There exists $q^{\prime} \in G / H$ such that:

$$
f\left(q^{\prime}\right)+\varphi(e H)>b .
$$

Let $g^{\prime} \in G$ such that $g^{\prime}$ projects to $q^{\prime}$ under the natural map $G \rightarrow G / H$. Set

$$
h(x)=f(x)+\left(L_{g^{\prime}} \varphi\right)(x)=f(x)+\varphi\left(g^{\prime-1} x\right) .
$$

Now for $x \notin g^{\prime-1}(C), h(x) \leq b$. Thus $h$ attains its maximum for some $q \in g^{\prime-1}(C)$. Hence

$$
(\Delta h)(q) \leq 0
$$

So

$$
0 \geq(\Delta f)(q)+\Delta\left(L_{g^{\prime}} \varphi\right)(q)=(\Delta f)(q)+(\Delta \varphi)\left(g^{\prime-1} q\right)
$$

and

$$
(\Delta f)(q) \leq-(\Delta \varphi)\left(g^{\prime-1} q\right) \leq\|\Delta \varphi\|<\varepsilon .
$$

Proposition 2.1. a) Suppose $f \in \mathscr{B}^{2}(G / H)$ and $\Delta f=\lambda f$. If $\lambda>0$, then $f \equiv 0$. b) Suppose $\Delta$ is the Laplacian of a $G$-invariant metric on $G / H$ and $f \in \mathscr{B}^{2}(G / H, C)$ and $\Delta f=\lambda f$. If $\operatorname{Re} \lambda>0$, then $f \equiv 0$.

Proof. a) From Lemma 2.1, there exist sequences of points $p_{n}, q_{n}$ in $G / H$ and a sequence of $\varepsilon_{n}>0$ such that:

(i) $\lim _{n \rightarrow \infty} f\left(q_{n}\right)=\sup f, \lim _{n \rightarrow \infty} f\left(p_{n}\right)=\inf f$,

(ii) $(\Delta f)\left(q_{n}\right)<\varepsilon_{n}, \Delta f\left(p_{n}\right)>-\varepsilon_{n}$, and

(iii) $\lim _{n \rightarrow \infty} \varepsilon_{n}=0$.

As $\Delta f=\lambda f, \lambda f\left(q_{n}\right)<\varepsilon_{n}$ and $\lambda f\left(p_{n}\right)>-\varepsilon_{n}$ and hence $\lambda \sup f \leq 0$ and $\lambda \inf f \geq 0$. As $\lambda>0,0 \geq \inf f \geq \sup f \geq 0$ and $f \equiv 0$.

b) Suppose $f(x)=u(x)+i v(x)$ and let $h(x)=|f(x)|^{2}$. Then from Proposition 2.1 there exist a sequence of points $q_{n}$ and a sequence $\varepsilon_{n}>0$ such that

(i) $\lim _{n \rightarrow \infty} h\left(q_{n}\right)=\sup h$,

(ii) $(\Delta h)\left(q_{n}\right)<\varepsilon_{n}$, and

(iii) $\lim _{n \rightarrow \infty} \varepsilon_{n}=0$.

Let $\langle$,$\rangle denote the G$-invariant Riemannian metric on $G / H$. Then

$$
\begin{aligned}
(\Delta h)(x)= & (\Delta f)(x) f(x)+f(x) \Delta f(x)+2\langle\operatorname{grad} u, \operatorname{grad} u\rangle_{x} \\
& +2\langle\operatorname{grad} v, \operatorname{grad} v\rangle_{x} \geq(\Delta f)(x) f(x)+f(x) \Delta f(x) \\
= & 2 \operatorname{Re} \lambda|f(x)|^{2} .
\end{aligned}
$$


Thus $\varepsilon_{n} \geq \Delta h\left(q_{n}\right) \geq 2 \operatorname{Re} \lambda\left|f\left(q_{n}\right)\right|^{2}$ and $0 \geq \sup h$. As $h \geq 0, h \equiv 0$ and therefore $f \equiv 0$. q.e.d.

This generalizes a result of E. B. Dynkin [1] on symmetric spaces.

Lemma 2.2. a) If $f \in \mathscr{B}^{2}(G / H)$ and $\lambda \geq 0$, then $\|(\Delta-\lambda) f\| \geq \lambda\|f\|$. b) If $f \in \mathscr{B}^{2}(G / H, C), \Delta$ is the Laplacian of a $G$-invariant Riemannian metric on $G / H$, and $\lambda \geq 0$, then $\|(\Delta-\lambda) f\| \geq \lambda\|f\|$.

Proof. a) Suppose $\|f\|=\sup f$. Select a sequence $q_{n}$ in $G / H$ and a sequence $\varepsilon_{n}>0$ such that:

(i) $\lim _{n \rightarrow \infty} f\left(q_{n}\right)=\sup f$,

(ii) $(\Delta f)\left(q_{n}\right)<\varepsilon_{n}$, and

(iii) $\lim _{n \rightarrow \infty} \varepsilon_{n}=0$.

Then $(\Delta-\lambda) f\left(q_{n}\right) \leq \varepsilon_{n}-\lambda f\left(q_{n}\right)$, and hence

$$
\inf (\Delta-\lambda) f \leq-\lambda \sup f=-\lambda\|f\| .
$$

Thus $\|(\Delta-\lambda)(f)\| \geq \lambda\|f\|$. If $\|f\|=-\inf f$, the proof is similar. Part b) is proved by setting $h(x)=|f(x)|^{2}$ and proceeding as above.

Lemma 2.3. Let $v_{n}, n \geq 1$, be a sequence of functions in $C^{2}(G / H)$ converging uniformly on compact subsets to 0 , and suppose $\Delta v_{n}$ converges uniformly on compact subsets to $f$. Then $f \equiv 0$.

Proof. Suppose not. Then we may assume that there is an open set $U$ with compact closure such that $f \mid U>B>0$. Without loss of generality we may assume $\sup _{x \in U}\left|v_{n}(x)\right| \leq 1 / 2^{n}$.

Let $V \subset U$ be an open set such that $\bar{V} \subset U$, and $\phi \in C_{0}^{\infty}(V)$ be such that $\phi \geq 0, \sup \phi=1$, and $|\Delta \phi| \leq C$. Then $v_{n}+\phi / 2^{n-1}$ attains a local maximum at some point $x_{n} \in V$. Thus

$$
\Delta v_{n}\left(x_{n}\right)+\left(1 / 2^{n-1}\right) \Delta \phi\left(x_{n}\right) \leq 0
$$

Hence

$$
\Delta v_{n}\left(x_{n}\right) \leq\left(1 / 2^{n-1}\right) C
$$

and for all $n$ we has $x_{n} \in V$ satisfying the above inequality. This contradicts the uniform convergence of $\Delta v_{n}$ on $\bar{V}$ to $f$. q.e.d.

Thus, if $g_{n}, n \geq 1$, is a sequence in $C^{2}(G / H), g_{n}$ converges uniformly on compact sets to $g$, and $\Delta g_{n}$ converges uniformly on compact sets to $f$, then we may say $\Delta g=f$. From now on we shall identify $\Delta$ with this extended operator.

We now consider the problem of solving the equation $(\Delta-\lambda) g=f$ for $f \in \mathscr{B}(G / H)$ and $\lambda>0$ with $g \in \mathscr{B}(G / H)$.

Proposition 2.2. Let $f \in \mathscr{B}(G / H)$ and $\lambda>0$. Then there exists $g \in \mathscr{B}(G / H)$ such that $(\Delta-\lambda) g=f$.

Proof. Suppose first that $f$ is $C^{\infty}$. It is clear that we may assume $f \geq 0$. 
Put on $G / H$ a complete $C^{\infty}$-Riemannian metric. Let $\Omega_{1} \subset \Omega_{2} \subset \cdots \subset \Omega_{n} \subset \ldots$ be a sequence of open subsets of $G / H$ with smooth boundaries such that $\bar{\Omega}_{n}$ is compact, $\bar{\Omega}_{n} \subset \Omega_{n+1}$, and $G / H=\bigcup_{n=1}^{\infty} \Omega_{n}$. Let $L$ be the Laplacian with respect to this metric, and $D_{t}=t(\Delta-\lambda)+(1-t) L$, and consider the elliptic boundary problem:

$D_{t} u=F$ on $\bar{\Omega}_{n}$ and $u=f$ on $\partial \Omega_{n}$ for $F \in C^{\infty}\left(\bar{\Omega}_{n}\right)$ and $f \in C^{\infty}\left(\partial \Omega_{n}\right)$. As ind $L$ $=0\left[4\right.$, p. 264] we see that ind $D_{t}=0$ for all $t$, and therefore that ind $(\Delta-\lambda)$ $=0$. If $u \in C^{\infty}\left(\bar{\Omega}_{n}\right)$, and $u=0$ on $\partial \Omega_{n}$, we have as in Lemma 2.1 that $\sup _{x \in \Omega_{n}}|(\Delta-\lambda) u(x)| \geq \lambda \sup _{x \in \Omega_{n}}|u(x)|$. Thus $\operatorname{ker}(\Delta-\lambda)=0$. Hence we may find a unique $u_{n} \in C^{\infty}\left(\bar{\Omega}_{n}\right), u_{n}=0$ in $\partial \Omega_{n}$, such that $(\Delta-\lambda) u_{n}=f$ in $\bar{\Omega}_{n}$. Set $u_{n}=0$ on the complement of $\Omega_{n}$.

Consider the functions $v_{n}=u_{n+1}-u_{n}$ and $u_{n}$. On the complement of $\Omega_{n}$ we have that $v_{n} \leq 0$ and $u_{n} \leq 0$. We claim that $v_{n} \leq 0$ and $u_{n} \leq 0$ everywhere. Otherwise, we can find points $x_{0}, y_{0} \in \Omega_{n}$ such that $0<v_{n}\left(x_{0}\right)=\sup v_{n}(x)$, and $0<u_{n}\left(y_{0}\right)=\sup u_{n}(x)$. However, we must have $\Delta v_{n}\left(x_{0}\right) \leq 0$ and $\Delta u_{n}\left(y_{0}\right) \leq 0$, but in $\Omega_{n}$ we have that $0 \geq \Delta v_{n}\left(x_{0}\right)=\lambda v_{n}\left(x_{0}\right)>0$ which is a contradiction, and $0 \geq \Delta u_{n}\left(y_{0}\right)=f\left(y_{0}\right)+\lambda u_{n}\left(y_{0}\right)>0$ which is also a contradiction. Thus $u_{n} \leq 0$ and $u_{n+1} \leq u_{n}$ for all $n$.

Since the $u_{n}$ 's form a bounded monotone sequence of functions, $\lim _{n \rightarrow \infty} u_{n}=g$ exists in the distribution topology on $G / H$. Hence $(\Delta-\lambda) g=f$ as distributions. As $f$ is $C^{\infty}$ and $\Delta$ has $C^{\infty}$-coefficients, we have that $g$ is $C^{\infty}$ and $(\Delta-\lambda) g=f$ as functions. Moreover, as $\left\|u_{n}\right\| \leq\|f\| / \lambda$ for all $n,\|g\| \leq\|f\| / \lambda$.

Suppose now only that $f \in \mathscr{B}(G / H)$. Select a sequence $f_{n} \in \mathscr{B}(G / H), n \geq 1$, such that $f_{n}$ is $C^{\infty}$ for all $n$ and $f_{n}$ converges to $f$ in $\mathscr{B}(G / H)$. Then there exists a sequence $g_{n} \in \mathscr{B}(G / H), n \geq 1$, such that $g_{n}$ is $C^{\infty}$ for all $n$ and $(\Delta-\lambda) g_{n}=f_{n}$. By Lemmas 2.2 and 2.3, $g_{n}$ converges to a $g$ in $\mathscr{B}(G / H)$, where $(\Delta-\lambda) g=f$.

Remark. The proof of the above proposition is a simplification of the original proof. Its improvement rests on an observation which was pointed out to the author by the referee.

We may now apply the Hille-Yosida theorem to obtain that for $\lambda>0$, $(1-\Delta / \lambda)^{-1}$ is a continuous operator of norm 1 on $\mathscr{B}(G / H)$,

$$
T_{t}=\exp t \Delta=\lim _{n \rightarrow \infty}(1-t \Delta / n)^{-n}
$$

defines a continuous operator of norm 1 on $\mathscr{B}(G / H)$ which commutes with the left action of $G$, and finally, for $f \in \mathscr{B}^{2}(G / H)$,

$$
\lim _{t \rightarrow 0} \frac{\left(T_{t}-1\right)}{t} f(x)=(\Delta f)(x)
$$

Let $\Phi_{t}(f)=\left(T_{t} f\right)(e)$. Then $\Phi_{t}$ is a continuous functional of norm 1 on 
$\mathscr{B}(G / H)$, and thus defines a measure on $\mathscr{B}(G / H)$. Note that $\Phi_{t}(c)=c$ for a constant $c$.

Lemma 2.4. If $f \in \mathscr{B}(G / H)$ and $f \geq 0$, then $\Phi_{t}(f) \geq 0$.

Proof. Suppose $\Phi_{t}(f)=-d<0$ and let $c=\|f\|$. Then $c-f \geq 0$ and $\|c-f\| \leq c$, but $\Phi_{t}(c-f)=c+d>c$ which is a contradiction. q.e.d.

Thus

$$
\Phi_{t}(f)=\int_{\beta(G / H)} f(x) d p_{t}(x),
$$

where $p_{t}$ is a probability measure on $\beta(G / H)$.

Now as $T_{t}\left(L_{g} f\right)=L_{g}\left(T_{t} f\right)$, we have that

$$
T_{t} f(g)=\Phi_{t}\left(L_{g-1} f\right)=\int_{\beta(G / H)}\left(L_{g-1} f\right)(x) d p_{t}(x)=\int_{\beta(G / H)} f(g x) d p_{t}(x) .
$$

From $T_{t} \cdot T_{s} f=T_{t+s} f$, it follows that

$$
\begin{aligned}
T_{t+s}(f)(g) & =\int_{\beta(G / H)} f(g x) d p_{t+s}(x)=T_{t}\left(T_{s} f\right)(g) \\
& =\int_{\beta(G / H)}\left(T_{s} f\right)(g y) d p_{t}(y)=\int_{\beta(G / H)} \int_{\beta(G / H)} f(g y x) d p_{s}(x) d p_{t}(y)
\end{aligned}
$$

Hence $p_{t}{ }^{*} p_{s}=p_{t+s}$, and the $p_{t}$ 's form a semigroup of probability measures on $\beta(G / H)$.

We now summarize our results.

Theorem 2.1. The $T_{t}$ 's for $t>0$ form a semigroup of continuous operators on $\mathscr{B}(G / H)$, which commute with the left action of $G$, and determine probability measures $p_{t}$ for $t>0$ on $\beta(G / H)$, which form a semigroup under convolution. Moreover, if $f \in \mathscr{B}(G / H)$, then

$$
T_{t}(f)(g)=\int_{\beta(G / H)} f(g x) d p_{t}(x) .
$$

\section{References}

[1] R. Courant \& D. Hilbert, Methods of mathematical physics, Vol. II, Interscience, New York, 1962.

[2] E. B. Dynkin, Brownian motion in certain symmetric spaces and nonnegative eigenfunctions of the Laplace-Beltrami operator, Amer. Math. Soc. Transl. (2) 72 (1968) 203-228.

[3] S. Helgason, Differential geometry and symmetric spaces, Academic Press, New York, 1962.

[ 4 ] L. Hörmander, Linear partial differential operators, Academic Press, New York, 1963. 
[ 5 ] G. A. Hunt, Semi-groups of measures of Lie groups. Trans. Amer. Math. Soc. 81 (1956) 264-293.

[6] H. Omori, Isometric immersions of Riemannian manifolds, J. Math. Soc. Japan 19 (1967) 205-214.

[7] R. Palais et al., Seminar on the Atiyah-Singer index theorem, Annals of Math. Studies, No. 57, Princeton University Press, Princeton, 1965.

[ 8 ] F. Reisz \& B. Sz-Nagy, Functional analysis, Ungar, New York, 1955.

RUTGERS UNIVERSITY 